\title{
X-Ray Spectra of Superheavy and Quasi-Superheavy Elements*
}

\author{
B. Fricke and J. T. Waber \\ Department of Materials Science, Northwestern University, Evanston, Illinois 60201
}

(Received 9 June 1972)

\begin{abstract}
We discuss the possibility of identifying superheavy elements from the observation of their $M$-shell x-ray spectra, which might occur during the collision of a superheavy element with a heavy target. The same question is discussed for the possible observation of the $x$ rays from the quasimolecule (quasi-superheavy element) which is formed during such a heavy-ion collision. It is shown that it is very difficult, if not impossible, to determine any information about the interesting quantum electrodynamical effects from the $M$-shell x-ray spectra of these quasimolecules.
\end{abstract}

\section{INTRODUCTION}

In a recent letter Armbruster, Mockler, and Stein ${ }^{1}$ discussed an identification scheme for superheavy elements. The identification is based on the observation of characteristic $M$-shell x rays which are expected to be produced in the superheavy element after collision with a heavy target. They base this prediction on a quasimolecular diagram ${ }^{2}$ which shows that two $3 d_{5 / 2}$ electrons from the superheavy element will be promoted during the collision process to such small energies that these electrons will be easily ionized. Therefore, after the collision, a hole will be transferred into the $3 d$ shell which will lead to observable $M$-shell x-ray radiation. Although we agree that during such a collision process $M$-shell x rays will be produced, we would like to point out the weakness of the argument of Armbruster et al. for element $Z=199$.

In part I of the paper we will briefly discuss the problems which arise and the approximations one can use when trying to get reliable predictions of physical quantities of the heavy superheavy elements, such as their energy eigenvalues. In part II we discuss the determination of the atomic number of a superheavy element and how reliable it can be if only $M$-shell $x$ rays are observable during such a collision experiment. In part III we will indicate how extensively energy levels are shifted by changes in the degree of ionization and the number of protons in the nucleus. In part IV we discuss the similar question of how much physical information might be extracted from the observation of $x$ rays which are emitted from the quasimolecule species itself.

Using a phenomenological description of one of the quantum electrodynamical effects, namely the vacuum fluctuation, we have been able to carry out calculations beyond element E175 with some credibility. ${ }^{3}$ With such energy eigenvalues of element E184 obtained in this manner we have drawn a correlation diagram (Fig. 1) which would result from the collision of E110 with $\mathrm{W}$.

In a diabatic correlation diagram ${ }^{2}$ such as Fig. 1 , levels are joined with the same symmetry $\boldsymbol{\Omega}$ along the internuclear line and the same number of radial nodes $n-|\kappa|$. When two nuclei collide the electrons may experience a nearly central field for a short time, perhaps $10^{-19} \mathrm{sec}$. Because of the restrictions just cited, certain levels are raised in energy [for example, $\left(3 d_{5 / 2}\right) \rightarrow$ nodeless $\left(5 g_{7 / 2}\right)$ level]. Thus some electronic levels remain unoccupied for the "compound" or quasimolecular atom.

From Fig. 1, it is obvious that all $M$ electrons of the element $E 110$ can be transferred into strongIy bound states and no promotion occurs because the $5 g$ level occurs at energies more negative than $-1 \mathrm{keV}$. Because of the expected very high degree of ionization of the quasimolecule itself, the $5 \mathrm{~g}$ level may very well be bound more tightly at -3 or $-4 \mathrm{keV}$. This would reduce the ionization probability of these electrons significantly. Therefore, a much larger fraction of the electrons which are promoted into the $5 \mathrm{~g}$ level of the quasi-superheavy element will return to the original $3 d$ level of the superheavy element. During the separation of the nuclei a hole might be transferred into the $3 d_{5 / 2}$ level of the superheavy element because of the large number of level crossings which occur when the two nuclei separate again. Therefore the cross section for observing $\boldsymbol{M}$-shell x rays from the superheavy element will be much smaller than anticipated by Armbruster et al.

Betz and co-workers ${ }^{4,5}$ have examined the motion of heavy ions such as uranium, bromine, and iodine ions accelerated to energies as large as $0.18 \mathrm{GeV}$ colliding with targets. The electrons which are removed depend on the ratio of their orbital velocity $\nu_{0}$ to the velocity $\nu$ of the projectile ion. An additional correction for the atomic number leads to a reduced velocity $\left(\nu / \nu_{0} Z^{2 / 3}\right)$. Thus it is unlikely that deeply lying core electrons 
will be removed. This means that $K$ or $L \mathrm{x}$-ray lines are unlikely to be emitted when the two nuclei continue past each other during collision, i.e., when the superheavy quasimolecular species is breaking up.

Betz et al. ${ }^{5}$ have shown that the ionization state after such a collision can often best be described as having most of the outermost electrons ionized. Thus single holes in inner shell might not occur and lead to such $\mathrm{x}$-ray lines. Also the chance for creation of two or more inner holes is very small.

A different argument might be given for there being some chance of creating $M$-shell $\mathrm{x}$ rays during the collision. In many cases, the main part of the cross section for producing $x$ rays can be described by the Coulomb excitation mechanism. One such example is given by Schnopper $e t a l .^{6} \mathrm{~A}$ very rough argument may be made. The $3 d$ electrons, for example, in element E114 are bound by $6 \mathrm{keV}$ with a radius of about 0.09 a.u. Electrons with a comparable binding energy and radius are very well known to be removed in collisions between heavy ions with lower $Z$. Hence we may assume that in a real process of a collision of a superheavy element with a heavy target, $M$-shell $x$ rays will indeed be produced.

\section{PREDICTIONS OF ENERGY EIGENVALUES FOR VERY HEAVY SUPERHEAVY ELEMENTS}

Any extrapolation of nonrelativistic physical quantities, such as energy eigenvalues, ${ }^{7}$ into the region of very superheavy elements can easily lead to wrong results. This has been established with a large number of relativistic Dirac-Fock and Dirac-Slater ${ }^{9}$ calculations that have been carried out up to $Z=172$, i.e., element E172. At element $\mathrm{E} 175$ these calculations reach a mathematical barrier because at this point the $1 s$ energy eigenvalues reach the negative continuum of the electrons (with a binding energy of $-2 m_{e} c^{2}$ ) and the integration procedure for the Dirac equation breaks down. In any calculation beyond this point, this problem can only be solved by including additional effects into the Hamiltonian. The first possible solution is to look into the physical problems which arise when a bound level becomes degenerate with those in the negative continuum, i.e., drops into the negative sea. Some statements about the coupling of electrons and positions coexisting in a strong field have been made by Akheizer and Berestetskii. ${ }^{10}$ Recently Müller et al. ${ }^{11}$ have shown that under some very simplifying assumptions, the wave function of an electron in the negative sea of electrons is localized, which would mean that the full number of electrons might be bound in such a very heavy superheavy element. No self-consistent field (SCF) calculation had been done which takes into account these electrons in the negative sea and hence no self-consistent energy eigenvalues are available for the $M$ and outer shells. Figure 1 is clearly more reliable than the nonrelativistic correlation diagram of Armbruster et al. for E199.

On the other hand, it is not at all clear whether the energy eigenvalues really drop into the negative sea of electrons when the atomic number exceeds 175 . It is possible that strong nonlinear electrodynamical ${ }^{12}$ or quantum electrodynamical ${ }^{3}$ effects might reduce the field and prevent this behavior. Were this assumption true, the usual type of SCF calculations could be controlled.

\section{IDENTIFICATION OF THE SUPERHEAVY ELEMENT FROM X RAYS AFTER THE COLLISION}

After having seen that we indeed may expect at least some $\boldsymbol{M}$-shell x rays from the superheavy element after a heavy-ion collision with a heavy target, we must turn to the question of whether these $x$ rays lead to a unique identification of the atomic number of the superheavy element. To discuss this quantitatively, we have listed in Table

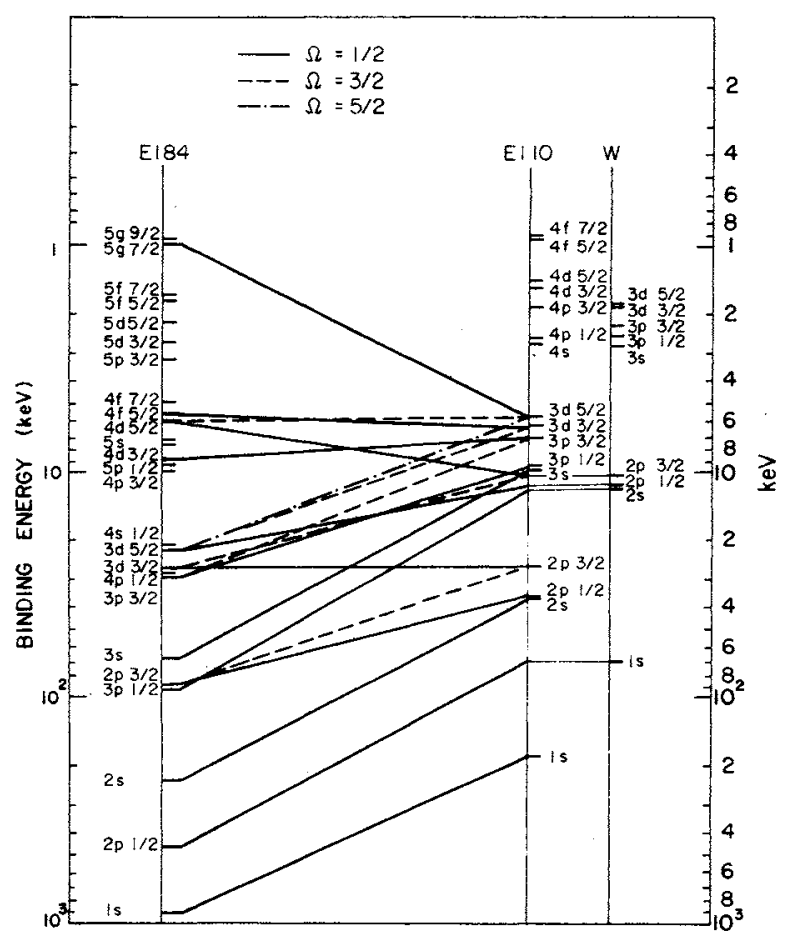

FIG. 1. Diabatic level diagram for the quasimolecule $W+E 110 . \Omega$ is the projection of the angular momentum $j$ on the internuclear axis. This quantity as well as the number of nodes of the electronic wave function has to be conserved during such a central collision. 
TABLE I. X-ray transition energies for various $K, L, M$, and $N$ lines in element E114. In column 2, the difference between the analogous transitions between elements E114 and E113 is given. The remaining columns list the change in transition energy due to various ionization states.

\begin{tabular}{|c|c|c|c|c|c|c|c|}
\hline \multirow[b]{2}{*}{ Transition } & \multirow{2}{*}{$\begin{array}{c}\boldsymbol{E}_{\gamma} \\
(\mathrm{keV})\end{array}$} & \multirow{2}{*}{$\begin{array}{c}E_{\gamma}(114)-E_{\gamma} \text { (113) } \\
(\mathrm{keV})\end{array}$} & \multicolumn{5}{|c|}{ Difference in $E_{\gamma}$ due to various ionization states in $\mathrm{eV}$} \\
\hline & & & $0^{+}-14^{+}$ & $0^{+}-22^{+}$ & $0^{+}-36^{+}$ & $0^{+}-46^{+}$ & $0^{+}-54^{+}$ \\
\hline $2 p_{3 / 2}-1 s_{1 / 2}$ & $\mathbf{1 7 2 . 2}$ & 4.26 & 0.1 & 1.0 & 0.2 & 0.5 & 5.2 \\
\hline $3 d_{5 / 2}-2 p_{3 / 2}$ & 21.8 & 0.42 & 3.3 & 9.3 & 4.4 & 34. & 71 \\
\hline $4 f_{7 / 2}-3 d_{5 / 2}$ & 5.35 & 0.114 & 2.5 & 7.0 & 37 & 80 & 125 \\
\hline $4 p_{3 / 2}-3 d_{5 / 2}$ & 4.29 & 0.091 & 2.6 & 6.9 & 42 & 89 & 137 \\
\hline $5 p_{3 / 2}-4 d_{5 / 2}$ & 1.11 & 0.032 & 3.5 & 13 & 80 & 160 & \\
\hline
\end{tabular}

I the total energies of some $K-, M-$, and $N$-shell $\mathrm{x}$ rays for element $\mathrm{E} 114$, the difference in energy of the analogous transtion for the neighboring element E113, as well as the expected difference in these transition energies for several ionization states. All values were taken from self-consistent relativistic Dirac-Slater ${ }^{5}$ calculations. We know that the absolute $x$-ray energies given in this Table I are expected to be correct only within 1 to $2 \%$ because all the relativistic corrections have not been included. This uncertainty does not, in principle, lead to any inaccuracy in the determination of the superheavy element. Two independent higher-accuracy calculations have been made recent$1 y^{13,14}$ for fermium $(Z=100)$ taking into account the magnetic, retardation, and the quantum electrodynamical corrections after making a self-consistent Dirac-Fock calculation. Analogous calculations for the $\mathrm{x}$ rays of superheavy elements near E114 are presently under investigation which means that the absolute $\mathrm{x}$-ray energies for a neutral atom or for a free +1 ion will be known in the near future with errors less than about $60 \mathrm{eV}$ for the $K \times$ rays and about $20 \mathrm{eV}$ for the $M$ x rays.

The remaining principal uncertainty about using the $\mathrm{x}$ rayc emitted after the collision with a heavy target for uniquely determining the atomic number is that one does not know the degree of ionization at the moment when the $x$ ray is emitted. The values in Table I show that a determination of the atomic number is very straightforward if $K$ or $L$ $x$ rays were available. However, the identification using only the $\boldsymbol{M}$-shell x rays is much more difficult. The difference between analogous x-ray lines of two neighboring elements is of the same order of magnitude as the difference caused by different degrees of ionization. The number of missing electrons $\bar{q}$ inside solid targets is not known very well. ${ }^{4}$ Thus the positive charge might be anywhere between 30 and 50 . But even the same degree of ionization does not lead to the same energy shift because the electron configuration of the excited states of ions is unknown. Different electron configurations may lead to further shifts of the lines of $M$-shell radiation by 80 to

\section{$100 \mathrm{eV}$.}

A unique $Z$ identification of superheavy elements by observing $M$-shell x rays after the collision with a heavy target will be relatively difficult. To make it possible, one needs experimental equipment with sufficiently high resolution to resolve the fine structure of the $M$ spectrum. In addition one needs in every case the theoretical help of relativistic SCF calculations plus the knowledge of all major corrections like vacuum fluctuation, vacuum polarization, magnetic and retardation interaction. Table I also shows very clearly that an identification using $N$-shell x rays will be impossible even if it would be possible to measure these $1.5-\mathrm{keV}$ lines very accurately.

\section{ENERGY LEVELS IN IONIZED SUPERHEAVY ELEMENTS}

The recent calculations of Nix and Fiset ${ }^{15}$ indicate that even if a new element E122 were formed by fusion of Ge and Th atoms after collision, the nuclear charge might rapidly be reduced to 114 or even 110 by emission of $\alpha$ particles. The correlation diagram of Fig. 2 has been modified to indicate the shift in energy of the electronic levels due to a change in nuclear charge as well as due to the change in the degree of ionization $\vec{q}$. The large shifts in the Dirac-Slater eigenvalues above reinforce the arguments given below in connection with the quasi-superheavy elements.

\section{ENERGY OF X RAYS FROM THE QUASI-SUPERHEAVY ELEMENTS}

As already pointed out, ${ }^{16}$ such a heavy-ion collision that proceeds with the formation of a quasicompound nucleus with a nearly central potential for the electrons may itself give rise to $\mathrm{x}$ rays from transitions which may occur during the very short lifetime of about $10^{-19} \mathrm{sec}$ of this system. It may very well be possible that a level with the principal quantum number $n=3$ in the quasisuperheavy element remains unoccupied in the first half of the collision process so that an $M$ - 
shell x-ray transition might occur. A further problem exists in addition to the fact that the probability for such a process will be very small. Such an $\mathrm{x}$-ray line emitted from the quasi-superheavy element will be broadened by several $\mathrm{keV}$ due to the uncertainty relation because the quasisuperheavy element exists only the very short time of about $10^{-19} \mathrm{sec}$.

The observation of the existence of such $x$-ray lines might itself be very interesting, but we think that very little useful information can be obtained. If the $M$-shell x-ray line results from the collision of two known heavy ions, the atomic number of the quasi-superheavy element is already known and thus it does not need to be extracted. If the atomic number of one of the heavy ions in the target is not known the identification of its $Z$ will be very questionable. Aside from the fact that we may not know the degree of ionization (and thus the associated shift of the $x$-ray lines of the quasisuperheavy element) it is more significant that we do not know the accurate absolute $x$-ray transition energies in the region of the very heavy superheavy elements. The reason is twofold.

First we have only rough estimations of the magnetic and retardation corrections as well as the quantum electrodynamical effects in this region. But even supposing we did use perturbation theory to calculate these corrections accurately, we would only get good energies for the $K$ - and probably the $L$-shell electrons. However, the wave functions of these inner electrons are altered by the electrodynamic effects and lead to screening of the electrons with $n>2$. We expect that the indirect relativistic effect would be larger than the direct effect. As an example for this trend we have calculated the vacuum polarization in a lower $-Z$ element for the $3 p$ electrons and have obtained one sign for the shift in energy when it is calculated in a self-consistent way, and the opposite sign when compared with the perturbation calculation. This is the second reason why we do not have accurate absolute $x-$ ray transition energies for very heavy superheavy elements. To treat the various corrections in the correct self-consistent way is not yet possible.

Besides these major problems, there are two more fundamental questions. First, is the DiracFock description an adequate description for superheavy elements, and second, are there more additional effects which may change the binding energies, such as possible nonlinear electrodynamical effects which would lead to the breakdown of the superposition principle?

Actually, if $x$-ray lines are observed from the system which we call quasi-superheavy element, the nuclei are still separated by many tens of
Fermis. Even if we assume that this two-center problem of the quasimolecule is solved correctly, still the uncertainty due to the effect of the extended nuclei remains. To give a feeling for this effect, we have shown ${ }^{5}$ that a change of the nuclear radius of an element like E164 leads to shifts for the $K$ electron in the order of $30 \mathrm{keV}$. This effect will be smaller for the outer electrons.

In addition to all these theoretical uncertainties, the experiment will be complicated because the line width will be several $\mathrm{keV}$.

Altogether, this study definitely shows that a unique identification of the the atomic number of the combined quasi-superheavy element will be impossible to within only a few integers. Of course it might be valuable by itself if the atomic number of such a system in the region of say element E160 or higher could be established to within \pm 5 . But this also means that the suggestion of Armbruster $e t$ al. that the observation of such $\mathrm{x}$ rays could yield an experimental determination of all the known and unknown contributions to the energy levels is, however, very questionable.

There might be two ways which may increase the amount of information from $x$ rays originating from the quasi-superheavy elements. If it is possible to observe these $x$ rays from quasimolecules where both partners are known, then the syste-

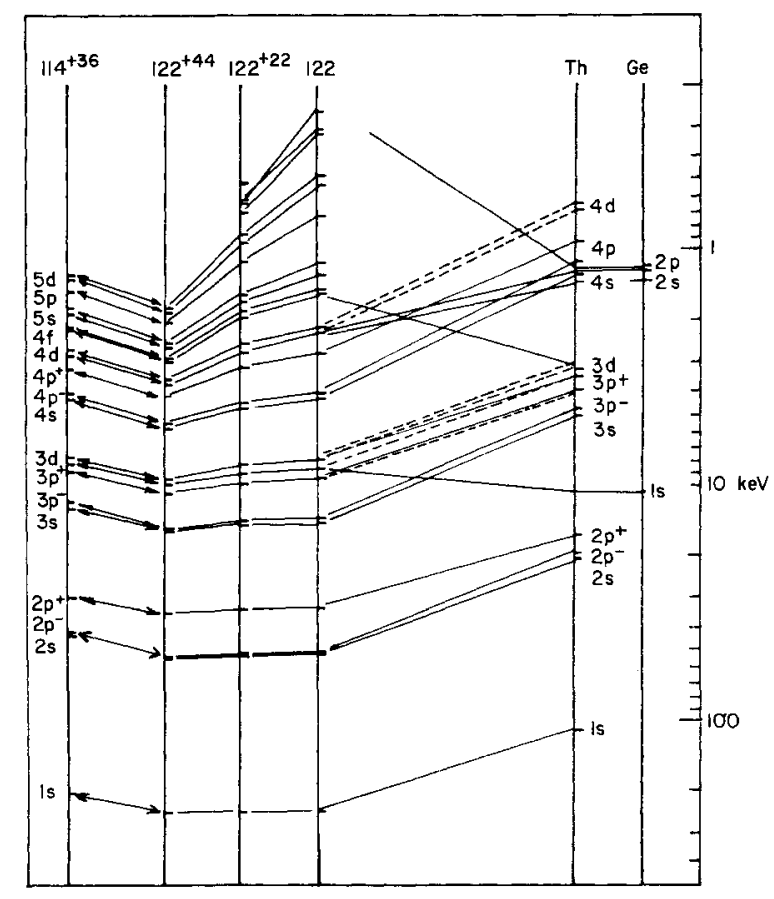

FIG. 2. Extended form of the diabatic correlation diagram for the uniting of Ge with Th atoms which illustrates the shift in orbital energy levels for loss of electrons and for the radioactive decrease in proton number of the nucleus. 
matic extrapolation of the $\mathrm{x}$-ray energies as functions of both partners may lead to better results; this is comparable with the systematic use of Mosley's diagram.

Secondly, measurement of the fine structure splitting may yield some more information, since the $s$ and $p_{1 / 2}$ electrons are influenced more strongly by the interesting various contributions like the quantum electrodynamical effects than the $p_{3 / 2}$ or the $d$ electrons. For the $M$ shell, for example, both $s$ and $p_{1 / 2}$ electrons are expected to be shifted in energy mainly due to the direct influence of these effects whereas the other electrons will be shifted in the opposite direction. This change in the fine structure compared with calculated and extrapolated values will give some additional information about the atomic number or the magnitude of the additional corrections.

\section{CONCLUSION}

The observation of $x$ rays emitted during and after the collision of a superheavy element (near E114) with a heavy target will be one of the interesting fields in the near future for heavy-ion physics. It seems to be possible to observe only $M$ - and higher-shell x-ray radiation from the superheavy element after the collision within the proposed experiment. This means that a unique identification of the atomic number of the superheavy element is already much more difficult than from the observation of $K$ or $L \mathrm{x}$ rays because of the unknown ionization state after the collision. But forthcoming absolute $x$-ray transition energy calculations for elements near E114 plus an increasing knowledge of the ionization states of the elements after the collision will make it possible even if no neighboring $x-r a y$ transition energies are available.

Any observation of $x$ rays from the quasi-superheavy element formed during the collision of two very heavy nuclei will itself be very interesting although any accurate identification of the atomic number of the quasi-superheavy element will not be possible. The same is true for the gain of knowledge of the interesting quantum electrodynamical effects in this region, at least if only $M$ shell $x$ rays can be observed. The only hope is that these effects become so strong in very heavy elements that the very large fine structure splitting may lead to some information about the influence of these effects on the energy eigenvalues. Of course, it would be much easier to obtain some information if one were able to find a way to measure the $L$ - or even better the $K$-shell x-ray energies.

As an over-all result, we are pessimistic since many difficulties in extracting and interpreting the information which one would like to obtain have not yet been overcome. In order to tackle this problem from an adequate theoretical point of view, it is necessary to write an SCF program which is able to take the several possible large influences on the binding energies of the inner electrons directly into account. One step in this direction has been taken ${ }^{3}$; in a model calculation, we have looked at the influences of these effects on the valence electronic configuration of very high $-Z$ superheavy elements.

Note added in proof: Evidence for quasi-superheavy atoms in the range $Z=132$ to 145 has been published by P. Mokler, H. Stein, and P. Armbruster, Phys. Rev. Lett. 29, 827 (1972).

\footnotetext{
*Work supported by a grant from the U. S. Atomic Energy Commission.

${ }^{1}$ P. Armbruster, P. H. Mokler, and H. J. Stein, Phys. Rev. Letters 27, 1623 (1971).

${ }^{2}$ W. Lichten, Phys. Rev. 164, 131 (1967).

${ }^{3}$ B. Fricke and J. T. Waber, J. Chem. Phys. 57, 371 (1972).

${ }^{4} \mathrm{H}$. D. Betz and L. Grodzins, Phys. Rev. Letters 25, 211 (1970).

${ }^{5}$ H, D. Betz, J. P. Dalvaille, K. Kalata, H. W. Schnopper, A. R. Sohval, K. W. Jones, and H. E. Wagner, Conference on Inner Shell Ionization Phenomena, Atlanta, Georgia, April 1972 (unpublished).

${ }^{6}$ H. W. Schnopper, A. R Sohval, H. D. Betz, J. P. Dalvaille, K. Kalata, K. W. Jones, and H. E. Wagner, Conference on Inner Shell Ionization Phenomena, Atlanta, Georgia, April 1972 (unpublished).
}

\footnotetext{
${ }^{7}$ F. Herman and S. Skillman, Atomic Structure Calculations (Prentice Hall, Englewood Cliffs, N. J., 1963).

${ }^{8} \mathrm{~J}$. B. Mann, in Proceedings of the Robert A. Welch Foundation XIII. The Transuranium Elements (The Robert A. Welch Foundation, Houston, Texas, 1969), p. 430 ; R. A. Penneman, J. B. Mann, and C. K. Jørgensen, Chem. Phys. Lett. 8,321 (1971).

${ }^{9}$ B. Fricke, W. Greiner, and J. T. Waber, Theor. Chim. Acta 21, 235 (1971); B. Fricke and J. T. Waber, Actinides Rev. 1, 433 (1971).

${ }^{10}$ A. I. Akhiezer and V. B. Berestetskii, Quantum Electrodynamics (Inter science, New York,1965), pp. 120,195ff.

${ }^{11}$ B. Müller, H. Peitz, J. Rafelski, and W. Greiner, Phys. Rev. Letters 28, 1235 (1972).

${ }^{12}$ J. Rafelski, L. P. Fulcher, and W. Greiner, Phys. Rev, Letters 27, 958 (1971).

${ }^{13}$ J. P. Desclaux, B. Fricke, and J. T. Waber, Phys.
} 
Rev. Letters 28, 714 (1972).

${ }^{14}$ M. S. Freedman, F. T. Porter, and J. B. Mann, Phys. Rev. Letters 28,712 (1972).

${ }^{15}$ E. O. Fiset and J. R. Nix, Los Alamos Scientific Laboratory Report No. LA-DC-13345, March 1972 (un- published).

${ }^{16}$ This problem has been discussed during recent years but the possibility of observation of $x$-ray lines from those quasi-superheavy elements was first pointed out in formal discussion by W. Greiner. 